\title{
Short distance QCD contribution to the electroweak mass difference of pions
}

\author{
A. A. Natale* \\ Instituto de Física Teórica, Universidade Estadual Paulista, Rua Pamplona 145, 01405-900 São Paulo, Brazil
}

(Received 14 January 1997)

\begin{abstract}
It is known that the short distance QCD contribution to the mass difference of pions is quadratic on the quark masses, and irrelevant with respect to the long distance part. It is also considered in the literature that its calculation contains infinities, which should be absorbed by the quark mass renormalization. Following a prescription by Craigie, Narison, and Riazuddin of a renormalization-group-improved perturbation theory to deal with the electromagnetic mass shift problem in QCD, we show that the short distance QCD contribution to the electroweak pion mass difference (with $m_{u}=m_{d} \neq 0$ ) is finite and, of course, its value is negligible compared to other contributions. [S0556-2821(97)03111-1]

PACS number(s): 13.40.Dk, 14.40.Aq
\end{abstract}

Recently there has been a lot of interest in the mass differences of pions and kaons. There are many reasons for this. These mass differences are important because they provide relations among the light quark masses, as well as they are a quite nice example of strong isospin breaking. But most of the renewed interest lies mainly in the possibility of applying the latest techniques to handle the strong interaction physics of the simplest hadrons. A very complete and detailed calculation of these mass differences has recently been performed by Donoghue and Pérez [1], and this work contains most of the relevant references up to now on this subject.

As explained in Ref. [1], the pion electromagnetic mass difference amplitude receives different contributions from the long and short distance QCD, with the first one dominating the calculation, and the second one canceling out at zeroth and first order in the quark masses. The short distance QCD contribution to the pion mass difference (proportional to the quark masses) was not calculated in Ref. [1]. It was correctly neglected because it is quadratic in the quark masses. However, when we look to the many references discussing this problem out of the chiral limit, i.e., taking into account a nonzero quark mass, which is important for the kaon mass difference (see, for instance, Ref. [2]), we note the comment that this calculation gives an infinite result, which must be renormalized by a quark mass counterterm. Actually, it is generally agreed that the short distance QCD part of the pion mass difference is small, and as far as we know we cannot find in the literature a precise numerical evaluation of its finite part. It seems also that in the most recent papers about mass differences, the weak interaction contribution has not been included and, although small, it is as important as the electromagnetic one when computing the high energy part of the mass splitting, because it modifies the convergence of the calculation.

It is the purpose of this work to show, according to a renormalization-group-improved perturbation theory prescription by Craigie, Narison, and Riazuddin [3], to deal with the electromagnetic mass shift in QCD that the short distance QCD contribution to the "electroweak" pion mass difference away from the chiral limit is "finite," contrary to what

*Electronic address: natale@axp.ift.unesp.br is assumed in the literature, and to calculate its value, which is indeed quite small even compared with the uncertainty in the long distance contribution.

It is opportune to consider first why the electromagnetic mass difference of hadrons (in particular, the proton-neutron mass difference) is supposed to have a divergence, and how the authors of Ref. [3] solved an apparent puzzle on this subject. Afterwards, considering the prescription of Ref. [3], the calculation of the electroweak mass difference of pions follows straightforwardly. The argument that the electromagnetic proton-neutron mass difference is divergent is established using the Cottingham formula for the mass difference [4]:

$$
\delta m_{h} \propto \int \frac{d Q^{2}}{Q^{2}} T_{\mu}^{\mu}(Q, P),
$$

where $T_{\mu \nu}(q, p)$ is the virtual forward Compton amplitude for the scattering of a virtual photon of momentum $q$ on a target of momentum $p\left(Q^{2}=-q^{2}\right.$ and $\left.P^{2}=-p^{2}\right)$. From the operator-product expansion (OPE) we know that the leading operator contributing to $T_{\mu}^{\mu}$ is $m_{f} \bar{\psi}_{f} \psi_{f}$, where $m_{f}$ and $\psi_{f}$ are quark masses and fields. Since this quantity is a renormalization-group invariant, $T_{\mu}^{\mu}$ behaves as a constant at large $q^{2}$, and the integral diverges. The puzzle that we referred to above appears when, based on the use of Schwinger-Dyson equations, it was affirmed that Eq. (1) is finite under certain conditions [5], i.e., the Compton amplitude would be given by the running quark masses, and the mass difference reads

$$
\delta m_{h} \propto \int_{Q_{0}^{2}}^{\infty} \frac{d Q^{2}}{Q^{2}} m_{f}\left(Q^{2}\right),
$$

where $Q_{0}^{2}$ is a cutoff above which we can use OPE and perturbation theory, and

$$
m_{f}\left(Q^{2}\right) \equiv m_{0 f}\left[\alpha_{s}\left(Q^{2}\right)\right]^{\gamma}
$$

where $m_{f}\left(Q^{2}\right)$ is the running mass, $\alpha_{s}\left(Q^{2}\right)$ is the strong running coupling constant, and $\gamma=12 /\left(33-2 n_{f}\right)$, where $n_{f}$ 
is the number of flavors. Equation (2) is finite as long as $n_{f} \geqslant 11$. The explanation of these conflicting results was presented in Ref. [3].

The authors of Ref. [3] noted that the results of Eq. (1) and Eq. (2) are different because they depend on the order of integration of the strong and electromagnetic corrections. It is obvious that the result should not depend on which interaction is considered first, and Craigie, Narison, and Riazuddin proposed a unique prescription that renders the mass shift calculation independent of the order of integration, as it must be. The technique, called a renormalization-group-improved perturbation theory, is implied in the use of the running quark mass in the calculation [3]. It was observed that there is nothing special about the finite result of Eq. (2) for $n_{f}>11$, except that a subset of diagrams in the renormalization-group-improved theory is finite, and when $n_{f}<11$ the regularization scheme of Ref. [3] for the mass shift gives the analytic continuation of the finite calculation. We will not repeat all the details of Ref. [3], and in the following we simply use their prescription. At this point it is almost trivial to foresee our result: the pion mass difference would diverge similarly to the proton-neutron mass difference. However, the QCD contribution to the first one is quadratic in the running quark masses instead of the linear dependence in the proton-neutron mass difference. Therefore, the integral of the mass shift has a faster convergence and is finite for $n_{f} \geqslant 5$.

To calculate the short distance QCD contribution to the electroweak pion mass difference we follow closely the procedure of Ref. [6]. We start with the propagator $\psi^{(i)}\left(q^{2}\right)$ of the electroweak covariant divergences of the hadronic currents $A^{\mu(i)}$ [where $i=1+\imath 2(3)$ stands for the charged (neutral) axial-vector pion current]:

$$
\psi^{(i)}\left(q^{2}\right)={ }_{l} \int d^{4} x e^{\imath q \cdot x}\left\langle 0\left|T D_{\mu} A^{\mu(i)}(x) D_{\nu} A^{\nu(i)+}(0)\right|\right\rangle,
$$

which, by PCAC (partial conservation of axial-vector current), may also be written as

$$
\begin{gathered}
\psi^{(1+12)}\left(q^{2}\right) \approx \frac{2 f_{\pi^{+}}^{2} m_{\pi^{+}}^{4}}{-q^{2}+m_{\pi^{+}}^{2}}, \\
\psi^{(3)}\left(q^{2}\right) \approx \frac{f_{\pi^{0} m_{\pi^{0}}^{4}}^{2}}{-q^{2}+m_{\pi^{0}}^{2}} .
\end{gathered}
$$

Developing Eq. (4), and equalizing it to Eq. (5) at $q=0$, we obtain (see Ref. [6])

$$
\begin{aligned}
2 f_{\pi}^{2}\left(m_{\pi^{+}}^{2}-m_{\pi^{0}}^{2}\right) \approx & e^{2} \int \frac{d^{4} q}{(2 \pi)^{4}}\left[D_{\mu \nu}^{\gamma}(q)-D_{\mu \nu}^{Z}(q)\right] \\
& \times\left[2 \Pi_{V}^{\mu \nu(3)}(q)-\Pi_{A}^{\mu \nu(1+\imath 2)}(q)\right],
\end{aligned}
$$

where $D_{\mu \nu}^{\gamma}\left(D_{\mu \nu}^{Z}\right)$ is the photon (weak neutral boson) propagator and $\Pi_{V(A)}^{\mu \nu(i)}$ are the two-point functions of the vector and axial-vector currents, which can be decomposed as

$$
\Pi^{\mu \nu}\left(q^{2}\right)=-\left(g^{\mu \nu}-\frac{q^{\mu} q^{\nu}}{q^{2}}\right) \Pi^{1}\left(q^{2}\right)+\frac{q^{\mu} q^{\nu}}{q^{2}} \Pi^{0}\left(q^{2}\right) .
$$

Equation (6) contains only the leading contributions to the electroweak pion mass difference. We neglected a term originated from the difference of quark condensates $\left(m_{u}-m_{d}\right)\langle\bar{u} u-\bar{d} d\rangle$, since the condensates cancel out at leading order. We also neglected a large part of the weak interaction contribution, which disappears in the limit $m_{u}=m_{d}$ [6]. This simplifying limit is assumed throughout our calculation. Finally, the scalar boson terms have also been dropped out. All these contributions to the short distance pion mass difference will be even smaller than the one we are considering. Inserting Eq. (7) into Eq. (6) and working in the Landau gauge we arrive at

$$
\begin{aligned}
2 f_{\pi}^{2}\left(m_{\pi^{+}}^{2}-m_{\pi^{0}}^{2}\right) \approx & 3 \imath e^{2} \int \frac{d^{4} q}{(2 \pi)^{4}}\left(\frac{1}{q^{2}}-\frac{1}{q^{2}-M_{Z}^{2}}\right) \\
& \times\left[2 \Pi_{V}^{1(3)}\left(q^{2}\right)-\Pi_{A}^{1(1+\imath 2)}\left(q^{2}\right)\right] .
\end{aligned}
$$

To evaluate the electroweak mass difference of pions we separate the integral of Eq. (8) into two pieces. From 0 to $Q_{0}^{2}$, corresponding to the long distance contribution, we can saturate the $\Pi$ s by the low-energy resonances $\rho$ and $A_{1}$, obtaining the classical result of Das et al. [7]. All the details of the calculation up to now can be found in Ref. [6]. The remaining part is the short distance contribution, where the $\Pi$ s can be calculated through perturbative QCD, and is the one in which we are interested. From the results of Ref. [8] we can determine the QCD prediction for the difference of the two-point functions of Eq. (8), which (at dominant order in the quark mass) is

$$
2 \Pi_{V}^{1(3)}\left(q^{2}\right)-\Pi_{A}^{1(1+\imath 2)}\left(q^{2}\right) \approx \frac{3}{8 \pi}\left(m_{u}+m_{d}\right)^{2},
$$

where $m_{u}$ and $m_{d}$ are the $u$ and $d$ quark masses. Here we differ from Ref. [6] and consider the prescription of Craigie, Narison, and Riazuddin [3], introducing the running and not the bare masses in the calculation of Eq. (8). We obtain the following integral for the short distance contribution to the mass difference:

$$
\begin{aligned}
2 f_{\pi}^{2}\left(m_{\pi^{+}}^{2}-m_{\pi^{0}}^{2}\right)_{\mathrm{SD}} \approx & \frac{9}{8 \pi} l e^{2} \int_{Q_{0}^{2}}^{\infty} \frac{d^{4} q}{(2 \pi)^{4}} \frac{-M_{Z}^{2}}{q^{2}\left(q^{2}-M_{Z}^{2}\right)} \\
& \times\left[m_{u}\left(q^{2}\right)+m_{d}\left(q^{2}\right)\right]^{2} .
\end{aligned}
$$

In the above expression, if we define $\Delta_{\mathrm{SD}} \equiv\left(m_{\pi^{+}}\right.$ $\left.-m_{\pi^{0}}\right)_{\mathrm{SD}}$, take $m_{\pi^{+}}+m_{\pi^{0}} \sim 2 m_{\pi}$, go to the Euclidean space and, as discussed previously, assume $m_{u}=m_{d}$ $=m_{f}\left(Q^{2}\right)$, we obtain

$$
\Delta_{\mathrm{SD}} \approx \frac{9 \alpha}{32 \pi^{2}} \frac{M_{Z}^{2}}{f_{\pi}^{2} m_{\pi}} \int_{Q_{0}^{2}}^{\infty} d Q^{2} \frac{m_{f}^{2}\left(Q^{2}\right)}{Q^{2}+M_{Z}^{2}}
$$

where $\alpha$ is the electromagnetic coupling constant. Note that the integral of Eq. (11) is similar to the ones discussed in Ref. [3], the integrand is proportional to $\ln ^{-2 \gamma}\left(Q^{2}\right) / Q^{2}$, and $\Delta_{\mathrm{SD}}$ is finite as long as $2 \gamma>1$, or $n_{f} \geqslant 5$. The fact that the 
two-point function difference in Eq. (9) cancels out at zeroth and first order in the quark masses is fundamental for this convergence.

The calculation of the integral in Eq. (11) is straightforward [3], and it is necessary to verify that it does lead to a negligible contribution. Its evaluation gives

$$
\Delta_{\mathrm{SD}} \approx-\frac{9 \alpha}{32 \pi^{2}} \frac{m_{0 f}^{2} Q_{0}^{2}}{f_{\pi}^{2} m_{\pi}}\left(\ln \frac{Q_{0}^{2}}{\Lambda_{\mathrm{QCD}}^{2}}\right)^{-2 \gamma} .
$$

Assuming $m_{0 f} \sim 10 \mathrm{MeV}$, and $Q_{0}^{2} \sim 1 \mathrm{GeV}^{2}$ as a good scale above which we can apply perturbative QCD, we obtain $\Delta_{\mathrm{SD}} \approx-0.02 \mathrm{MeV}$ which is a value smaller than the uncertainty present in the long distance contribution to the pion mass difference, and perfectly negligible. Unfortunately the short distance behavior of the mass difference $\left(\Delta m_{\pi}\right)$ turned out to be highly dependent on the cutoff $Q_{0}^{2}$, and we must have $\partial \Delta m_{\pi} / \partial Q_{0}^{2}=0$, reflecting the independence of the physical result on the choice of this separation scale. However, we can expect that with a better knowledge of the tran- sition between the nonperturbative and perturbative regions this abrupt behavior will be softened.

In conclusion, we have computed the short distance QCD contribution to the electroweak pion mass difference out of the chiral limit (assuming $m_{u}=m_{d}$ ), following a prescription of Craigie, Narison, and Riazuddin. We have shown that this mass difference is finite and, as expected, is totally negligible compared to the long distance contribution. Although the numerical result just serves to corroborate how negligible it is, we have not seen the arguments of Ref. [3] applied to the pion mass difference before. Moreover, it is important to stress the effect of the remaining standard model interactions when discussing the high energy part of these mass differences, because the convergence of the result is affected by these interactions.

I am grateful for discussions with P. S. Rodrigues da Silva. This research was supported in part by the Conselho Nacional de Desenvolvimento Científico e Tecnológico (CNPq).
[1] J. F. Donoghue and A. F. Pérez, this issue, Phys. Rev. D 55, 7075 (1997).

[2] J. Bijnens, Phys. Lett. B 306, 343 (1993).

[3] N. S. Craigie, S. Narison, and Riazuddin, Nucl. Phys. B174, 207 (1980).

[4] J. C. Collins, Nucl. Phys. B149, 90 (1979).

[5] S. J. Brodsky, G. F. de Téramond, and I. A. Schmidt, Phys.
Rev. Lett. 44, 557 (1980).

[6] B. Machet and A. A. Natale, Ann. Phys. (N.Y.) 160, 114 (1985).

[7] T. Das, G. S. Guralnik, V. S. Mathur, F. E. Low, and J. E. Young, Phys. Rev. Lett. 18, 759 (1967).

[8] E. G. Floratos, S. Narison, and E. de Rafael, Nucl. Phys. B155, 115 (1979). 